\title{
Ring Currents in Polycyclic Sodium Clusters
}

\author{
Slavko Radenković ${ }^{\mathrm{a}, \mathrm{b}}$ and Patrick Bultinck ${ }^{\mathrm{b}, *}$ \\ ${ }^{a}$ Faculty of Science, University of Kragujevac, P.O. Box 60, 34000 Kragujevac, \\ Serbia \\ ${ }^{\mathrm{b}}$ Department of Inorganic and Physical Chemistry, Ghent University, Krijgslaan 281 \\ (S3), 9000 Gent, Belgium.
}

In the recent work by Khatua et al. [Khatua, S.; Roy, D. R.; Bultinck, P.; Bhattacharjee, M.; Chattaraj, P. K. Phys. Chem. Chem. Phys. 2008, 10, 2461-2474] the synthesis and structure of a fac-trioxo molybdenum metalloligand and its sodium complex containing 1D hexagonal chains of sodium ions was reported. In the same paper, the aromaticity of hexagonal Na-clusters was quantified by means of the nucleus-independent chemical shift and electronic multicentre indices. It was shown that the aromaticity of hexagonal Na-clusters is of the same order as the aromaticity of analogous benzenoid hydrocarbons. In the present study current density maps are used to rationalize the aromaticity of polycyclic Na-clusters. It is shown that although polycyclic Na-systems sustain a diatropic ring current, the induced current density is several times weaker than in analogous benzenoid hydrocarbons. A detailed analysis indicates that the current density in hexagonal Na-systems is almost completely determined by four HOMO $\sigma$-electrons.

\section{INTRODUCTION}

After the discovery of the aromatic character of $\left[\mathrm{Al}_{4}\right]^{2-} /\left[\mathrm{Al}_{4}\right]^{4-}$ by Boldyrev et $a l^{1,2}$ the concept of aromaticity was extended from its usual organic realm to the field of all-metal inorganic clusters. Compared to the aromaticity encountered in organic molecules, primarily being of the traditional $\pi$-type, all-metal systems can exhibit a multifold aromaticity ${ }^{3-5}$ and conflicting aromaticity, ${ }^{2,3,6-9}$ arising from their $\sigma_{-}, \pi-, \delta-$ ${ }^{10,11}$ and even $\varphi^{-}{ }^{12}$ electron delocalization. Until recently, there was no report on the

\footnotetext{
" Corresponding author E-mail: Patrick.Bultinck@ugent.be
} 
synthesis and structural characterization of aromatic compounds containing alkali metal ions. Two reports ${ }^{13,14}$ dealt with the synthesis and structural characterization of a fac-trioxo molybdenum metalloligand and its sodium $\left[\mathrm{Na}_{2} \mathrm{MoO}_{3} \mathrm{~L}\left(\mathrm{H}_{2} \mathrm{O}\right)_{2}\right]_{\mathrm{n}}$ and potassium complex $\left[\mathrm{K}_{2} \mathrm{MoO}_{3} \mathrm{~L}\left(\mathrm{H}_{2} \mathrm{O}\right)_{3}\right]_{\mathrm{n}},\{\mathrm{L}=$ iminodiacetate $\}$. The analysis of the crystal structure of those compounds showed that the Na-complex contains linear chains of $\mathrm{Na}_{6}$ hexagons, whereas the K-complex contains $2 \mathrm{D}$ hexagonal chains. These distinctive features of $\mathrm{Na}$ - and K-complexes and their clear structural similarity with hydrocarbons were a motivation to investigate their aromaticity. The aromaticity of the polycyclic alkali clusters was previously ${ }^{14}$ quantified by means of the nucleusindependent chemical shift (NICS) ${ }^{15}$ and electronic multicentre indices (MCI). ${ }^{16}$ It was shown that individual $\mathrm{Na}_{6}$ rings are considerably more aromatic than the corresponding $\mathrm{K}_{6}$ rings. Furthermore, according to NICS values the aromaticity of Na-clusters is of the same order of magnitude as the aromaticity of linear polyacenes.

The importance of newly reported Na- and K-clusters can be viewed from different aspects. In recent years syntheses of $3 \mathrm{D}$ polymeric materials are receiving more and more attention due to their potential use as small molecule storage materials. ${ }^{17-19}$ On the other hand, both $\mathrm{Na}$ - and K-clusters contain all-metal rings stabilized with appropriate ligands, making them different from all-metal clusters containing 'bare' metal rings. ${ }^{1-5}$ The aromaticity of 'ligand stabilized' rings of metal atoms becomes a new emerging field of aromaticity.

The problem with claims that a certain species is aromatic is that this conclusion may depend rather significantly on the property used to characterize aromaticity. As an example: a ring that exhibits bond length equalization as in benzene but does not sustain a ring current could be classified by some as aromatic but by others as not aromatic depending on the relative importance they attach to the property of bond lengths equalization or ring currents respectively. The same is true for e.g., electron delocalization and ring currents. Although one of us has previously shown that electron delocalization and ring currents in carbohydrates go hand in hand, ${ }^{20-23}$ the more general and subtle interrelationship is that a delocalized system is a necessary but not sufficient requirement for a ring current. In previous work on $\mathrm{Na}_{6}$ systems, electron delocalization was gauged by the multicentre index and information on a ring current was obtained indirectly from NICS values. The problem with NICS, however, is that although usually a negative (or aromatic) NICS value reflects the 
existence of a ring current, there is no direct way to extract and thus beyond doubt prove the existence of an underlying ring current.

The direct visualization and thus proof of existence or non-existence of a ring current was much advanced by the work of Keith and Bader $^{24,25}$ with their introduction of a perturbation theory based scheme for computing induced current density maps with the so-called continuous set of gauge transformations. This method is now also known as the continuous transformation of origin of current density with diamagnetic zero CTOCD-DZ ${ }^{26-28}$ or ipsocentric method. ${ }^{29-31}$ These methods may be used to give a direct visualization of the induced current density in polycyclic Naclusters and their analogous linear polyacenes. The significant advantage of this method is that the total current density is naturally partitioned into molecular-orbital contributions, ${ }^{29-31}$ allowing an efficient rationalization of different types of aromaticity $(\sigma, \pi$, etc.). The results obtained by analyzing the current density are compared with calculated NICS values. The bond cross sections of the current density are used to analyze the nature and patterns of the current density induced in polycyclic Na-clusters and their analogous hydrocarbons. This way, we aim to establish the nature of the aromaticity in terms of sustaining of a ring current for the Na-clusters.

\section{COMPUTATIONAL METHODS}

The structures of the $\mathrm{Na}_{4 n+2}(n=1-5)$ entities (Figure1) were taken from the experimental structural data given by Khatua et al. ${ }^{13,14}$ The molecular structures of the linear polyacenes $\mathrm{C}_{4 n+2} \mathrm{H}_{2 n+4}(n=1-5)$ were optimized at the B3LYP/6-311+G* level. Computed Hessian matrices showed the optimized structures to correspond to minima on the potential energy surface.

The current density maps presented in this paper were computed by means of coupled HF theory using the diamagnetic-zero variant of the continuous transformation of origin of current density (CTOCD-DZ) method. ${ }^{26-28}$ In this method, the current density at each point in the molecule is computed by choosing itself as the origin of vector potential, hence the alternative name 'ipsocentric' for the method. ${ }^{29-31}$ The current density maps for $\mathrm{Na}_{4 n+2}$ and $\mathrm{C}_{4 n+2} \mathrm{H}_{2 n+4}(n=1-5)$ were calculated using the STO-3G, 3-21G and 6-31G Gaussian basis sets and for $n=1-3$ using the 6-31G* 
basis set. LANL2DZ calculations including the LANL2DZ effective core potential were carried out in order to be able to reduce the impact of the core electrons. In all calculations a unit magnetic field perpendicular to the molecular plane was used and the calculated ring currents were plotted on a grid in the plane parallel to the molecular plane with a diatropic current represented by a counterclockwise circulation.

The ring current maps for linear polyacenes were calculated $1 \mathrm{a}_{0}$ above the molecular plane, in accordance with the previous current maps calculations for such systems. $^{29,32}$

NICS $^{15}$ were calculated at the HF level through the gauge-including atomic orbital method (GIAO). ${ }^{33,34}$ The HF-method was used in order to make more reasonable the comparison of NICS values and the ring current results calculated at the same level. NICS(0)- and NICS(1)-values were calculated using all basis sets used for the ring current maps calculation (STO-3G, 3-21G, 6-31G, 6-31G*, LANL2DZ). Also, the basis set $6-311+\mathrm{G}^{*}$ was used in order to compare the HF/6-311+G* level NICS with the B3LYP/6-311+G* results from the previous work. ${ }^{14}$

The geometry optimization and calculation of NICS were done using Gaussian 03. ${ }^{35}$ Calculations of ring currents were performed using our own Fortran routines requiring as input formatted checkpoint files from Gaussian 03.

\section{RESULTS AND DISCUSSION}

As mentioned above, the structures of the different polycylic Na-systems were taken from experimental structural data of a fac-trioxo molybdenum metalloligand and complexes. ${ }^{13,14}$ It should be noted that these structures do not correspond to minima on the potential energy surfaces so although this paper shows their aromatic character in the compounds mentioned, no claim is made that aromaticity is the fundamental reason for the planar structure they exhibit in the compounds mentioned. The similarity between the Na-systems and linear polyacenes is clear although the symmetry of the compounds is not entirely the same. The $\mathrm{Na}_{6}$ compound for example, in the structure it is found to have experimentally, ${ }^{13,14}$ has a $\mathrm{D}_{2 \mathrm{~h}}$ symmetry rather than the $\mathrm{D}_{6 \mathrm{~h}}$ symmetry typical for benzene. 
Still, the structural similarity is sufficiently manifest to use the analogous linear polyacenes well-known as one of the most studied aromatic organic compounds $^{36-38}$, as a natural reference for the polycyclic Na-systems. NICS values calculated at the HF level for $\mathrm{Na}_{4 n+2}$ and $\mathrm{C}_{4 n+2} \mathrm{H}_{2 n+4}(n=1-5)$ are given in Table 1 and Table 2. By comparing the HF/6-311+G* with the B3LYP/6-311+G* results from Ref 14 it can be seen that both methods give very similar results, with a small difference that HF values for benzenoid polyacenes are somewhat bigger than those at the B3LYP level. By inspection of the data from Table 1, it is revealed that NICS-values do not depend too much on the basis set used. In the case of benzenoid hydrocarbons NICS values are more sensitive on the type of basis set (Table 2). Although, the STO$3 \mathrm{G}$ basis set gives the lowest NICS values for both Na-clusters and acenes, the results obtained using this basis set are in good qualitative agreement with the larger basis sets results. According to the NICS-values outer rings are less aromatic than the inner rings of Na-clusters. These findings are in complete analogy with the results for linear polyacenes, although this does not necessarily reflect larger local aromaticity in the inner rings. On the other hand, there is no analogy when NICS scans are in question. In the case of Na-clusters NICS-values monotonically become less negative as one moves from the molecular plane, whereas in the case of acenes NICS-values are the most negative at about $1 \AA$ above the molecular plane (Figure S1). Based on the similar results for the NICS scan and by examining orbital contributions to MCI it was concluded that Na-clusters are $\sigma$-aromatic. ${ }^{14}$

The map of the current density for the $\mathrm{Na}_{6}$-cluster calculated in the molecular plane using the $6-31 \mathrm{G}^{*}$ basis set is presented in Figure $2 \mathrm{a}$. It can be seen that there are strong local currents around the $\mathrm{Na}$ atoms, whereas other parts of the current are significantly weaker than the current density of benzene (Figure S5a). Analysis of the orbital contributions to the total current density in the $\mathrm{Na}_{6}$-clusters reveals that almost all significant contributions come from the circulation of $4 \sigma$-electrons from the two degenerate HOMOs. This finding is analogous to the well-known model predicting that only $4 \pi$-electrons significantly contribute to the total ring currents in benzenoid hydrocarbons. ${ }^{29}$ The current density contributions from the two degenerate HOMOs in the molecular plane of $\mathrm{Na}_{6}$ are shown in Figure $2 \mathrm{~b}$. It can be seen that the HOMOpair electrons in $\mathrm{Na}_{6}$ contribute clearly localized density currents rather than global circulations although a much weaker global current persists. 
In order to investigate the global current density of Na-clusters, first the dependence of the intensity of the induced current on the distance from the plane of $\mathrm{Na}_{6}$-ring was examined. Figure 3 shows the maximum current density $J_{\max }$ in the plotting plane parallel to the molecular plane as a function of the distance between both planes. $J_{\max }$, being the largest magnitude of current density in the plotting plane is a good indication of current strength. By comparing its value over a set of different planes parallel to the molecular plane, one can also locate the plane with the strongest current density. Figure 3 a clearly shows that the maximum in $J_{\max }$ in this plot occurs in the molecular plane. However, the observed current density and thus also the value of $J_{\max }$ is the result of a sum of all orbital contributions to the current density and its topology and features must not necessarily be due to the valence orbitals only. In order to establish the influence of the core electrons, the current densities were also calculated with the LANL2DZ basis set containing an effective core potential for the $\mathrm{Na}$ atoms, and thus removing the contributions from the $\mathrm{Na}$ core electrons. From Figure $3 \mathrm{~b}$ it can be seen that $J_{\max }$ is very strongly reduced in the molecular plane if we remove the core electrons. It can be observed that using the LANL2DZ basis set with the effective core potential causes the HOMOs to do not use basis functions corresponding to core electrons. Moreover, we also see that according to Figure $3 b$, $J_{\max }$ decreases fairly slowly upon increasing the distance between the plotting plane and the molecular plane. By comparison between $J_{\max }$ values in Figures $3 \mathrm{a}$ and $3 \mathrm{~b}$, it was established that for all electron basis sets, the most suitable plane is that at $1 \mathrm{a}_{0}$ above the molecular plane. The $J_{\max }$ values at $1 \mathrm{a}_{0}$ above the molecular plane for an all-electron basis set tend to be very comparable to what is obtained in the plane when using the LANL2DZ basis set with effective core potential. The current density maps, with the LANL2DZ basis set and effective core potential, computed in the molecular plane and $1 \mathrm{a}_{0}$ above the molecular plane are practically identical (Figures S8 and S9) allowing us to also use the plane $1 \mathrm{a}_{0}$ above the molecular plane for this level of theory. It should be noted that despite working in a plane above the molecular plane, the fact that there is only a slow decay of $J_{\max }$ as shown in Figure $3 \mathrm{~b}$ still suggests that the current density is of $\sigma$ type. The reason is that the current density of $\sigma$ type does not necessarily have to drop rapidly which is in sharp contrast to a $\pi$ type current density that for symmetry reasons has a node in the molecular plane. 
The maps of the total current density and contributions from the HOMO pair in the $\mathrm{Na}_{6}$-cluster calculated $1 \mathrm{a}_{0}$ above the molecular plane using the $6-31 \mathrm{G}^{*}$ basis set are presented in Figure S7. From Figure S7, it can be seen that the induced ring current density is substantially weaker than in benzene (Figure S5a). With $J_{\max }=$ 0.014 a.u. (calculated at the CTOCD-DZ/HF/6-31G* level) the current density in the $\mathrm{Na}_{6}$-cluster is about seven times weaker than in benzene with $J_{\max }=0.098$ a.u. (calculated at the CTOCD-DZ/HF/6-31G*//B3LYP/6-311+G* level). In order to make the current density map from Figure S7 more convenient for further discussion the arrow size was increased by a factor 3 as in Figure $2 \mathrm{c}$ and $2 \mathrm{~d}$. These enlarged arrows are used to show the current density maps of Na-clusters in the rest of the paper. The ring current maps for the series of $\mathrm{Na}_{4 n+2},(n=1,5)$ calculated using the 6$31 \mathrm{G}$ basis set are given in Figure 4. Analogous maps for $\mathrm{C}_{4 n+2} \mathrm{H}_{2 n+4},(n=1,5)$ are given in Figure S4. All other maps of current density calculated with different basis sets are given in Figures S8-S12. By comparing these ring current maps it is obvious that the STO-3G basis set gives current density somewhat stronger than the current density calculated with the other basis sets. With $J_{\max }=0.032$ a.u. calculated for the $\mathrm{Na}_{6}{ }^{-}$ cluster using the STO-3G basis set, the current density obtained by using this basis set is about two times stronger than the corresponding current density calculated with the 6-31G* basis set $\left(J_{\max }=0.014\right.$ a.u.). However, the current density maps calculated with the STO-3G basis set are in good qualitative agreement with the larger basis set maps. This finding reinforces the well-established opinion that most often sufficiently accurate current density maps can be obtained using modest basis sets. ${ }^{32,39}$

From Figure 4 two characteristic features are immediately recognized. First, all examined Na-clusters sustain a diatropic ring current. Second, the induced current density of the Na-systems is several times weaker than in the analogous linear polyacenes. The plots from Figure 4 reveal further analogies between the Na-clusters and hydrocarbons. It can be seen that the intensity of the total ring current density increases from the outer to the central ring of the Na-clusters, whereas the induced current in the terminal rings decreases with the size of the given Na-system. The last two observations are completely in agreement with the predictions made by NICSvalues (Table 1 and 2).

The $\mathrm{Na}_{6}$ system, although computed here in the experimental $\mathrm{D}_{2 \mathrm{~h}}$ symmetry rather than the $\mathrm{D}_{6 \mathrm{~h}}$ symmetry known for benzene, can be regarded as an experimental realization of a pseudo- $\pi$ system. It has been known for a long time that a hexagonal 
arrangement of six hydrogen atoms bears a large similarity to benzene. ${ }^{40}$ This has led to the practice of removing hydrogen atoms in (large) hydrocarbons and replacing the carbon atoms by hydrogen atoms. For the resulting structure, the current density is computed using the STO-3G basis set. Experience has shown that this leads to very similar current density maps as when using the true molecule ${ }^{41,42}$ and even shows a remarkable numerical similarity for current densities but also NICS values and Multicenter Indices. ${ }^{21-23,43,44}$ The $\mathrm{Na}_{6}$ system thus can be considered to be a true experimental realization of a pseudo- $\pi$ system.

In the ipsocentric method, one can show that the current density in a molecule depends on three key elements. In the perturbation theory ansatz, one needs to have an appropriate combination of orbital symmetries with the symmetry corresponding to the angular and linear momentum operators. Next, the combination of an occupied and virtual orbital must be characterized by a sufficiently small energy difference. Finally, there should be sufficient overlap between the occupied and virtual orbital involved taking into account the appropriate operator (see Ref. 29 for details). In case of benzene the main contributor to current density comes from transitions between the HOMO and LUMO levels. In the $\mathrm{Na}_{6}$ system with the reduced symmetry a somewhat similar picture appears where transitions from the HOMO level to the near-degenerate LUMO level again play the most important role and all significant contributions reflect a diatropic transition. The transition diagram is shown in Figure 5.

Using the current density profiles Fliegl et $a .^{45}$ pointed out that the widespread notion that the ring current in benzene is transported by $\pi$-electrons on both sides of the ring should be checked and reexamined. In a recent study, Monaco et $a l{ }^{46}$ have used orbital contributions to the bond current strength ${ }^{47}$ to give a quantitative proof that the ring current of benzene is transported by $\pi$-electrons on both sides of the ring. Similarly, these conclusions can be obtained by analyzing the results shown in Figure 6. The results presented in Figure 6 are obtained at the CTOCD-DZ/HF/6-31G*//B3LYP/6-311+G* level. The plot $6 b_{1}$ shows the total current density cross sections for a plane perpendicular to the $\mathrm{C} 1-\mathrm{C} 2$ bond of benzene and passing through the bond centre. For details see the Figure 6 caption. The cross sections of the current density shown in Figure 6 are in good agreement with the cross sections reported in Ref. 45 and 46. Figure 6 also shows the dependence of the $y$ component of the current density vector $\left(J_{y}\right)$ on the distance from the ring centre along the $x$-axes in the plane of the benzene ring (Figure $6 b_{2}$ ) and $1 a_{0}$ above the benzene 
ring (Figure $6 b_{3}$ ). Similar plots are made for the $\sigma$ current density (Figure $6 c_{1}, 6 c_{2}$ and $6 c_{3}$ ) and for the $\pi$-current density (Figure $6 d_{1}, 6 d_{2}$ and $6 d_{3}$ ). Analyzing the plots $6 b_{2}$ and $6 b_{3}$ one sees that inside the benzene ring there is a paratropic current which comes completely from the $\sigma$-electrons. By comparing the values of $J_{y}$ from the plots $6 c_{2}$ and $6 c_{3}$ it can be seen that the paratropic current inside the benzene ring decreases along the z-direction, i.e. along the direction perpendicular to the molecular plane. It is also obvious that the current density in the molecular plane comes completely from $\sigma$-electrons (plots $6 b_{2}$ and $6 d_{2}$ ). From the plots $6 b_{3}, 6 c_{3}$ and $6 d_{3}$ one can see that there is a diatropic current on the outside of the molecular ring. A diatropic current in the molecular plane comes from the $\sigma$-electrons. When moving perpendicularly from the plane of benzene molecule, the contributions from $\sigma$-orbitals get smaller whereas the contributions from $\pi$-orbitals get bigger and dominant.

An analogous analysis of the CTOCD-DZ/HF/6-31G* current cross sections for the $\mathrm{Na}_{6}$-complex is presented in Figure 7. The plot $7 b_{1}$ shows the total current density cross sections for a plane perpendicular to the $\mathrm{Na} 1-\mathrm{Na} 2$ bond of $\mathrm{Na}_{6}$ and passing through the bond centre (see Figure $7 \mathrm{a}$ ). The plots $7 b_{2}$ and $7 b_{3}$ show the dependence of the $J_{y}$ on the distance from the ring centre along the $x$-axes in the plane of the $\mathrm{Na}_{6}$ ring and $1 \mathrm{a}_{0}$ above the $\mathrm{Na}_{6}$ ring. The analogous plots for the HOMO pair contributions to the total current density are shown in the plots $7 c_{1}, 7 c_{2}$ and $7 c_{3}$. It is obvious that the results for the total current and the results for the HOMO pair current are almost identical, indicating that the contributions from the HOMO pair to the total current density dominate the total current. These findings support the model that only the four HOMO $\sigma$-electrons determine almost completely the total current density. By comparing the plots $7 b_{2}$ and $7 b_{3}$ it can be seen that the intensity of the current density decreases with increasing the distance from the molecular plane, which is a typical feature of the $\sigma$-electrons currents. Also, it is obvious that the magnitudes of the current density in the molecular plane and $1 \mathrm{a}_{0}$ above the molecular plane are very similar. Figure 7 also shows that a diatropic current is transported on the both sides of the $\mathrm{Na}_{6}$ ring by the $\sigma$-electron circulation. Plots $7 d_{1}, 7 d_{2}$ and $7 d_{3}$ show analogous results for the current density coming from the HOMO-2 (see Figure S13). One can see that the HOMO-2 contributions to the total current density are an order of magnitude smaller than the corresponding contributions of the HOMO pair and that the HOMO-2 contributions are not relevant in the analysis of the total current. 


\section{CONCLUSIONS}

In the present study CTOCD-DZ current density maps were used to rationalize the aromaticity of polycyclic Na-clusters and analogous linear polyacenes. The NICS values were also calculated with the HF method through the gauge-including atomic orbital method (GIAO). According to NICS values Na-clusters have a similar degree of the aromaticity as the analogous acenes. On the other hand, it was shown that although polycyclic Na-systems sustain a diatropic magnetic-field induced ring current, the induced current density is several times weaker that in analogous benzenoid hydrocarbons. This finding is in agreement with the results obtained using the multicentre indices predicting that the aromaticity of individual $\mathrm{Na}_{6}$-rings is smaller than the aromaticity of analogous rings in benzenoid hydrocarbons. ${ }^{14} \mathrm{~A}$ detailed analysis indicates that the current density in sodium hexagonal systems is almost completely determined by four HOMO $\sigma$-electrons.

\section{ACKNOWLEDGEMENTS}

P.B. acknowledges the FWO-Vlaanderen for continuous support. All calculations were performed using the Stevin supercomputer infrastructure of Ghent University. S.R. acknowledges Ghent University for a Basileus post-doc fellowship. The authors thank Dr. Stijn Fias for assistance with plotting the induced current density maps.

\section{REFERENCES}

(1) Li, X.; Kuznetsov, A. E.; Zhang, H.-F.; Boldyrev, A. I.; Wang, L.-S. Sience 2001, 291, 859-861.

(2) Kuznetsov, A. E.; Birch, K.; Boldyrev, A. I.; Li, X.; Zhai, H.; Wang, L.-S. Sience 2003, 300, 622-625.

(3) Boldyrev, A. I.; Wang, L. -S. Chem. Rev. 2005, 105, 3716-3757.

(4) Tsipis, C. A. Coord. Chem. Rev. 2005, 249, 2740-2762. 
(5) Zubarev, D. Y.; Avekiev, B. B.; Zhai, H. -J.; Wang, L. -S.; Boldyrev, A. I. Phys. Chem. Chem. Phys. 2008, 10, 257-267.

(6) Havenith, R. W. A.; Fowler, P. W.; Steiner, E.; Shetty, S.; Kanhere, D.; Pal, S. Phys. Chem. Chem. Phys. 2004, 6, 285-288.

(7) Lin, Y.-C.; Jusélius, J.; Sundholm, D.; Gauss, J. J. Chem. Phys. 2005, 122, 214308.

(8) Islas, R.; Heine, T.; Merino, G. J. Chem. Theory. Comput. 2007, 3, 775781.

(9) Ugrinov, A.; Sen, A.; Reber, A. C.; Qian, M.; Khanna, S. N. J. Am. Chem. Soc. 2008, 130, 782-783.

(10) Zhai, H. J.; Averkiev, B. B.; Zubarev, D. Y.; Wang, L. -S.; Boldyrev, A. I. Angew. Chem. Int. Ed. 2007, 46, 4277-4280.

(11) Averkiev, B. B.; Boldyrev, A. I. J. Phys. Chem. A 2007, 111, 1286412866.

(12) Tsipis, A. C.; Kefalidis, C. E.; Tsipis, C. A. J. Am. Chem. Soc. 2008, 130, 9144-9155.

(13) Khatua, S.; Roy, D. R.; Chattaraj, P. K.; Bhattacharjee, M. Chem. Commun. 2007, 135-137.

(14) Khatua, S.; Roy, D. R.; Bultinck, P.; Bhattacharjee, M.; Chattaraj, P. K. Phys. Chem. Chem. Phys. 2008, 10, 2461-2474.

(15) Schleyer, P. v. R.; Maerker, C.; Dransfeld, A.; Jiao, H.; Hommes, N. J. R. v. E. J. Am. Chem. Soc. 1996, 118, 6317-6318.

(16) Bultinck, P.; Ponec, R.; Van Damme, S. J. Phys. Org. Chem. 2005, 18, 706-718.

(17) Liu, X.; Park, M.; Hongh, S.; Oh, M.; Yoon, J. W.; Chang, J. S.; Lah, M. S. Inorg. Chem. 2009, 48, 11507-11509.

(18) Ren, H.; Ben, T.; Wang, E.; Jing, X.; Xue, M.; Liu, B.; Cui, Y.; Qiu, X.; Zhu, G. Chem. Commun. 2010, 46, 291-293.

(19) Zou, R. -Q.; Jiang, L.; Senoh, H.; Takeichi, N.; Xu, Q. Chem. Commun. 2005, 3526-3528.

(20) Bultinck, P.; Fias, S.; Ponec, R. Chem. -Eur. J. 2006, 12, 8813-8818.

(21) Fias, S.; Van Damme, S.; Bultinck, P. J. Comput. Chem. 2008, 29, 358366. 
(22) Fias, S.; Fowler, P.; Delgado, J. L.; Hahn, U.; Bultinck, P. Chem. -Eur. J. 2008, 14, 3093-3099.

(23) Fias, S.; Van Damme, S.; Bultinck, P. J. Comput. Chem. 2010, 31, 22862293.

(24) Keith, T. A.; Bader, R. F. W. J. Chem. Phys. 1993, 99, 3669-3682.

(25) Keith, T. A.; Bader, R. F. W. Chem. Phys. Lett. 1993, 210, 223-231.

(26) Coriani, S.; Lazzeretti, P.; Malagoli, M.; Zanasi, R. Theor. Chim. Acta 1994, 89, 181-192.

(27) Lazzeretti, P.; Malagoli, M.; Zanasi, R. Chem. Phys. Lett. 1994, 220, 299304.

(28) Zanasi, R. J. Chem. Phys. 1996, 105, 1460-1469.

(29) Steiner, E.; Fowler, P. W. J. Phys. Chem. A 2001, 105, 9553-9562.

(30) Steiner, E.; Fowler, P. W. Chem. Commun. 2001, 2220-2221.

(31) Fowler, P. W.; Havenith, R. W. A. J. Phys. Chem. A 2002, 106, 70487056.

(32) Steiner, E.; Fowler, P. W. Int. J. Quantum Chem. 1996, 60, 609-616.

(33) Wolinski, K.; Hilton, J. F.; Pulay, P. J. Am. Chem. Soc. 1990, 112, 82518260.

(34) Cheeseman, J. R.; Trucks, G. W.; Keith, T. A.; Frish, J. M. J. Chem. Phys. 1996, 104, 5497-5509.

(35) Gaussian 03, Revision C.02, Frisch, M. J.; Trucks, G. W.; Schlegel, H. B.; Scuseria, G. E.; Robb, M. A.; Cheeseman, J. R.; Montgomery, J. A. Jr.; Vreven, T,; Kudin, K. N.; Burant, J. C.; Millam, J. M.; Iyengar, S. S.; Tomasi, J.; Barone, V.; Mennucci, B.; Cossi, M.; Scalmani, G.; Rega, N.; Petersson, G. A.; Nakatsuji, H.; Hada, M.; Ehara, M.; Toyota, K., Fukuda, R.; Hasegawa, J.; Ishida, M.; Nakajima, T.; Honda, Y.; Kitao, O.; Nakai, H.; Klene, M.; Li, X.; Knox, J. E.; Hratchian, H. P.; Cross, J. B.; Bakken, V.; Adamo, C.; Jaramillo, J.; Gomperts, R.; Stratmann, R. E.; Yazyev, O.; Austin, A. J.; Cammi, R.; Pomelli, C.; Ochterski, J. W.; Ayala, P. Y.; Morokuma, K.; Voth, G. A.; Salvador, P.; Dannenberg, J. J.; Zakrzewski, V. G.; Dapprich, S.; Daniels, A. D.; Strain, M. C.; Farkas, O.; Malick, D. K.; Rabuck, A. D.; Raghavachari, K.; Foresman, J. B.; Ortiz, J. V.; Cui, Q.; Baboul, A. G.; Clifford, S.; Cioslowski, J.; Stefanov, B. B.; Liu, G.; Liashenko, A.; Piskorz, P.; Komaromi, I.; Martin, R. L.; Fox, D. J.; Keith, T.; Al-Laham, M. A.; Peng, C. Y.; Nanayakkara, A.; 
Challacombe, M.; Gill, P. M. W.; Johnson, B.; Chen, W.; Wong, M. W.; Gonzalez, C.; Pople, J. A. Gaussian, Inc., Wallingford CT, 2004.

(36) Randić, M. Chem. Rev. 2003, 103, 3449 -3606.

(37) Balaban, A. T.; Schleyer, P. v. R.; Rzepa, H. S. Chem. Rev. 2005, 105, $3436-3447$.

(38) Cyrański, M. K. Chem. Rev. 2005, 105, 3773-3811.

(39) Ligabue, A.; Pincelli, U.; Lazzeretti, P.; Zanasi, R. J. Am. Chem. Soc. 1999, $121,5513-5518$.

(40) London, F. J Phys Radium 1937, 8, 397-409.

(41) Fowler, P. W.; Steiner, E. Chem. Phys. Lett. 2002, 364, 259-266.

(42) Monaco, G.; Viglione, R.G.; Zanasi, R.; Fowler, P.W. J. Phys. Chem. A 2006, $110,7447-7452$.

(43) Bultinck, P.; Mandado, M.; Mosquera, R. J. Math. Chem. 2008, 43, 111118.

(44) Fias, S.; Bultinck, P. Faraday Discuss. 2007, 135, 381-384.

(45) Fliegl, H.; Sundholm, D.; Taubert, S.; Jusélius, J.; Klopper, W. J. Phys. Chem. A 2009, 113, 8668-8676.

(46) Monaco, G.; Zanasi, R.; Pelloni, S.; Lazzeretti, P. J. Chem. Theory Comput. 2010, 6, 3343-3351.

(47) Jusélius, J.; Sundholm, D.; Gauss, J. J. Chem. Phys. 2004, 121, 3952-3963. 


\begin{tabular}{|c|c|c|c|c|c|c|c|c|}
\hline & \multicolumn{6}{|c|}{ Basis set } \\
\hline & & & I & II & III & IV & $\mathbf{V}$ & VI \\
\hline \multirow{18}{*}{$\stackrel{\infty 00}{\Xi}$} & \multirow{2}{*}{ A } & $\operatorname{NICS}(0)$ & -8.86 & -8.37 & -8.33 & -8.47 & -8.38 & -8.69 \\
\hline & & NICS(1) & -7.54 & -7.37 & -7.35 & -7.49 & -7.44 & -7.50 \\
\hline & \multirow{2}{*}{ B } & $\operatorname{NICS}(0)$ & -8.43 & -9.65 & -9.64 & -9.62 & -9.53 & -9.91 \\
\hline & & $\operatorname{NICS}(1)$ & -7.25 & -8.42 & -8.43 & -8.43 & -8.38 & -8.52 \\
\hline & \multirow{2}{*}{ C } & $\operatorname{NICS}(0)$ & -7.70 & -9.25 & -9.29 & -9.24 & -9.14 & -9.54 \\
\hline & & $\operatorname{NICS}(1)$ & -6.64 & -8.05 & -8.10 & -8.08 & -8.01 & -8.19 \\
\hline & \multirow{2}{*}{ D } & $\operatorname{NICS}(0)$ & -9.14 & -11.39 & -11.39 & -11.31 & -11.20 & -11.34 \\
\hline & & $\operatorname{NICS}(1)$ & -7.94 & -9.89 & -9.90 & -9.86 & -9.80 & -9.76 \\
\hline & \multirow{2}{*}{$\mathbf{E}$} & $\operatorname{NICS}(0)$ & -7.20 & -8.19 & -8.28 & -8.21 & -8.10 & -8.58 \\
\hline & & $\operatorname{NICS}(1)$ & -6.20 & -7.10 & -7.19 & -7.15 & -7.07 & -7.33 \\
\hline & \multirow{2}{*}{$\mathbf{F}$} & $\operatorname{NICS}(0)$ & -8.91 & -11.71 & -11.73 & -11.65 & -11.53 & -11.68 \\
\hline & & $\operatorname{NICS}(1)$ & -7.74 & -10.17 & -10.20 & -10.15 & -10.08 & -10.05 \\
\hline & \multirow{2}{*}{$\mathbf{G}$} & $\operatorname{NICS}(0)$ & -6.90 & -6.57 & -6.78 & -6.68 & -6.55 & -7.07 \\
\hline & & $\operatorname{NICS}(1)$ & -5.94 & -5.65 & -5.85 & -5.78 & -5.68 & -5.99 \\
\hline & \multirow{2}{*}{$\mathbf{H}$} & $\operatorname{NICS}(0)$ & -8.58 & -11.15 & -11.20 & -11.09 & -10.97 & -11.21 \\
\hline & & $\operatorname{NICS}(1)$ & -7.46 & -9.65 & -9.71 & -9.64 & -9.57 & -9.61 \\
\hline & \multirow{2}{*}{ I } & $\operatorname{NICS}(0)$ & -9.01 & -13.07 & -13.05 & -12.97 & -12.86 & -12.97 \\
\hline & & $\operatorname{NICS}(1)$ & -7.84 & -11.38 & -11.37 & -11.33 & -11.28 & -11.19 \\
\hline
\end{tabular}

Table 1. NICS(0) and NICS(1) values of individual rings of Na-clusters depicted in Figure 1. NICS values were calculated at HF level using the STO-3G (I), 3-21G(II), 6-31G(III), 6-31G*(IV), 6-311+G*(V) basis set and the LANL2DZ basis set with effective core potential (VI). 


\begin{tabular}{|c|c|c|c|c|c|c|c|c|}
\hline & \multicolumn{6}{|c|}{ Basis set } \\
\hline & & & I & II & III & IV & $\mathbf{V}$ & VI \\
\hline \multirow{18}{*}{$\stackrel{\infty}{\leftrightarrows}$} & \multirow{2}{*}{$\mathbf{A}$} & $\operatorname{NICS}(0)$ & -9.09 & -9.96 & -8.58 & -11.53 & -9.49 & -7.88 \\
\hline & & $\operatorname{NICS}(1)$ & -10.20 & -12.56 & -11.98 & -12.82 & -11.25 & -11.21 \\
\hline & \multirow{2}{*}{ B } & $\operatorname{NICS}(0)$ & -8.18 & -10.03 & -8.66 & -11.33 & -9.60 & -7.80 \\
\hline & & $\operatorname{NICS}(1)$ & -9.83 & -12.63 & -12.00 & -12.88 & -11.46 & -10.95 \\
\hline & \multirow{2}{*}{ C } & $\operatorname{NICS}(0)$ & -6.71 & -8.61 & -7.23 & -9.53 & -7.92 & -6.49 \\
\hline & & $\operatorname{NICS}(1)$ & -8.71 & -11.34 & -10.70 & -11.42 & -10.08 & -9.74 \\
\hline & \multirow{2}{*}{ D } & $\operatorname{NICS}(0)$ & -12.02 & -14.37 & -13.16 & -16.03 & -14.56 & -12.20 \\
\hline & & $\operatorname{NICS}(1)$ & -12.21 & -15.81 & -15.09 & -16.14 & -14.82 & -13.75 \\
\hline & \multirow{2}{*}{$\mathbf{E}$} & NICS & -5.54 & -7.31 & -5.93 & -7.94 & -6.41 & -5.25 \\
\hline & & $\operatorname{NICS}(1)$ & -7.80 & -10.20 & -9.54 & -10.09 & -8.79 & -8.62 \\
\hline & \multirow{2}{*}{$\mathbf{F}$} & $\operatorname{NICS}(0)$ & -9.88 & -12.84 & -11.45 & -14.16 & -12.83 & -10.21 \\
\hline & & $\operatorname{NICS}(1)$ & -11.34 & -14.99 & -14.33 & -15.42 & -14.14 & -12.90 \\
\hline & \multirow{2}{*}{ G } & $\operatorname{NICS}(0)$ & -4.73 & -6.34 & -4.94 & -6.72 & -5.17 & -4.29 \\
\hline & & $\operatorname{NICS}(1)$ & -7.16 & -9.34 & -8.66 & -9.07 & -7.76 & -7.76 \\
\hline & \multirow{2}{*}{$\mathbf{H}$} & $\operatorname{NICS}(0)$ & -9.13 & -12.03 & -10.64 & -13.14 & -11.82 & -9.43 \\
\hline & & $\operatorname{NICS}(1)$ & -10.75 & -14.28 & -13.60 & -14.57 & -13.29 & -12.20 \\
\hline & \multirow{2}{*}{ I } & $\operatorname{NICS}(0)$ & -10.73 & -13.91 & -12.53 & -15.38 & -14.17 & -11.26 \\
\hline & & $\operatorname{NICS}(1)$ & -12.00 & -15.91 & -15.25 & -16.49 & -15.26 & -13.80 \\
\hline
\end{tabular}

Table 2. NICS(0) and NICS(1) values of individual rings of linear polyacenes analogous to Na-clusters depicted in Figure 1. NICS values were calculated at HF level using the STO-3G (I), 3-21G(II), 6-31G(III), 6-31G*(IV), 6-311+G*(V) basis set and the LANL2DZ basis set with effective core potential (VI). Geometries were optimized at the B3LYP/6-311+G* level. 

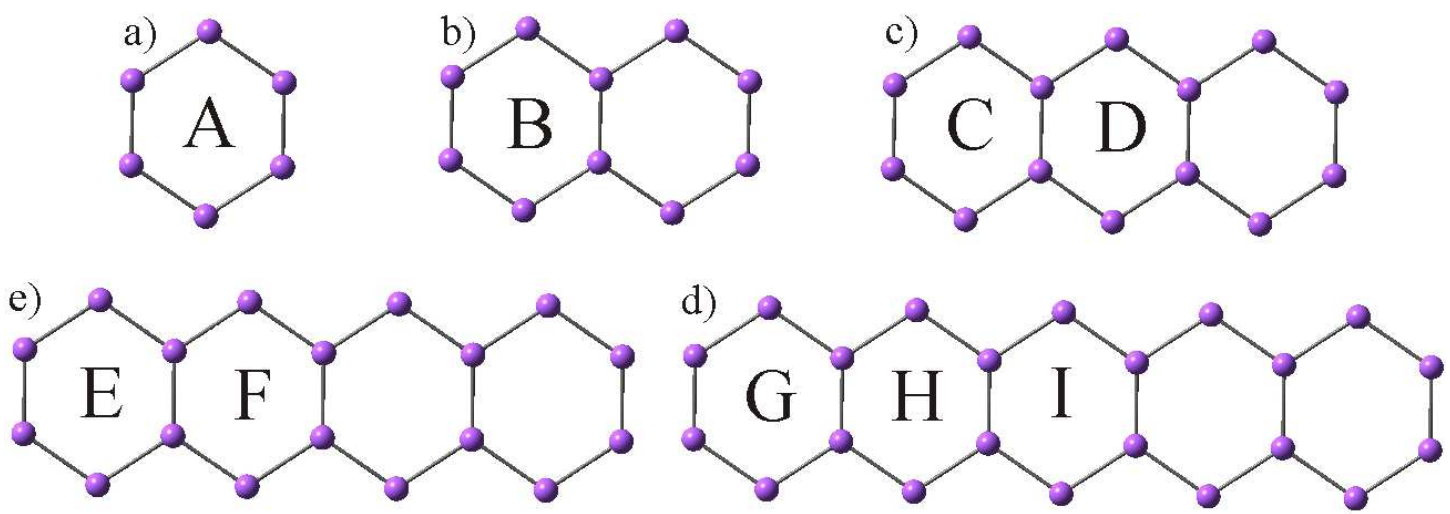

Figure 1. The structures of Na-clusters: a) $\mathrm{Na}_{6}$ b) $\mathrm{Na}_{10}$ c) $\mathrm{Na}_{14}$ d) $\mathrm{Na}_{18}$ e) $\mathrm{Na}_{22}$. The geometries of Na-clusters were taken from the experimental crystallographic data. ${ }^{14}$ The capital letters denote individual six-membered rings used for the NICS calculations. 

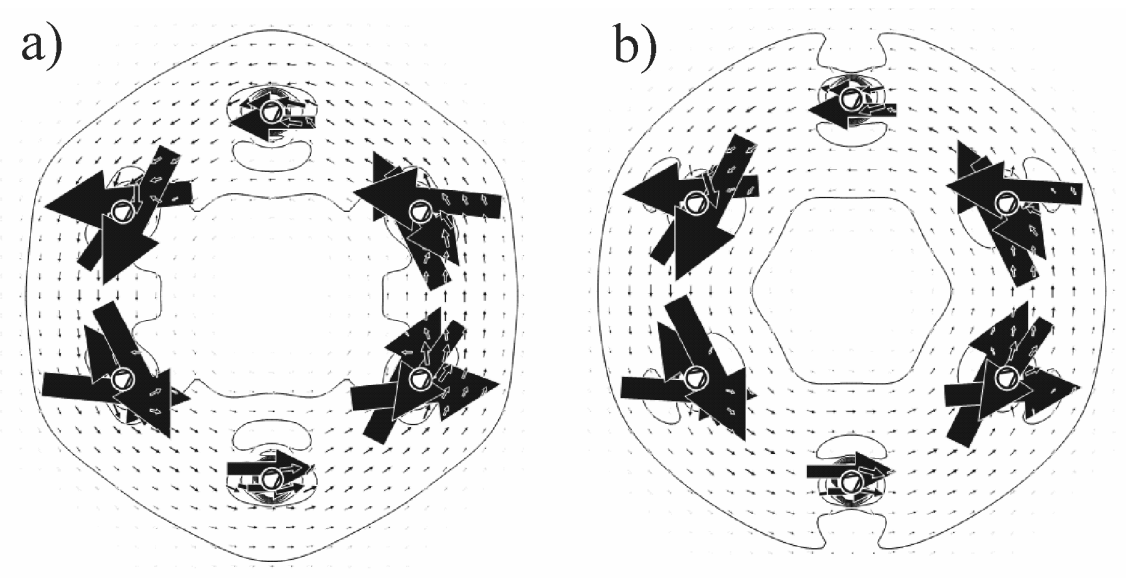

c)

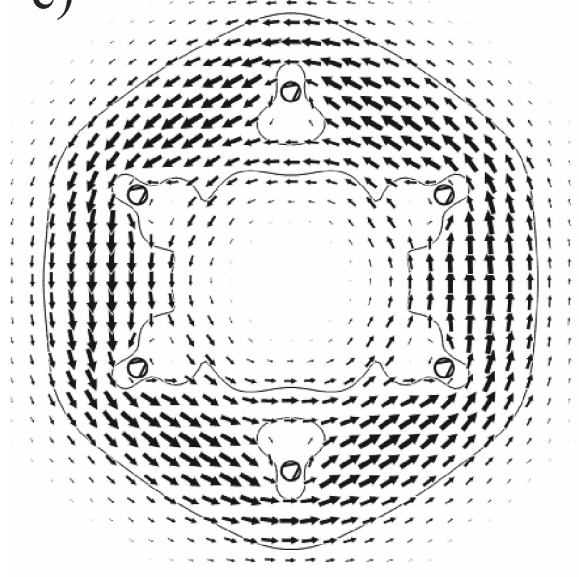

d)

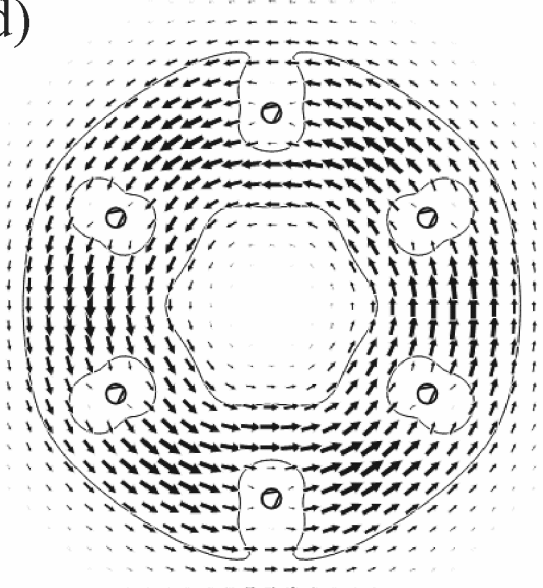

Figure 2. Current density maps of $\mathrm{Na}_{6}$ calculated using the 6-31G* basis set: a) total current density in the molecular plane, b) HOMO pair contributions to the total current density presented in a), c) total current density $1 \mathrm{a}_{0}$ above the molecular plane with the arrows three times enlarged, d) HOMO pair contributions to the total current density presented in c). 

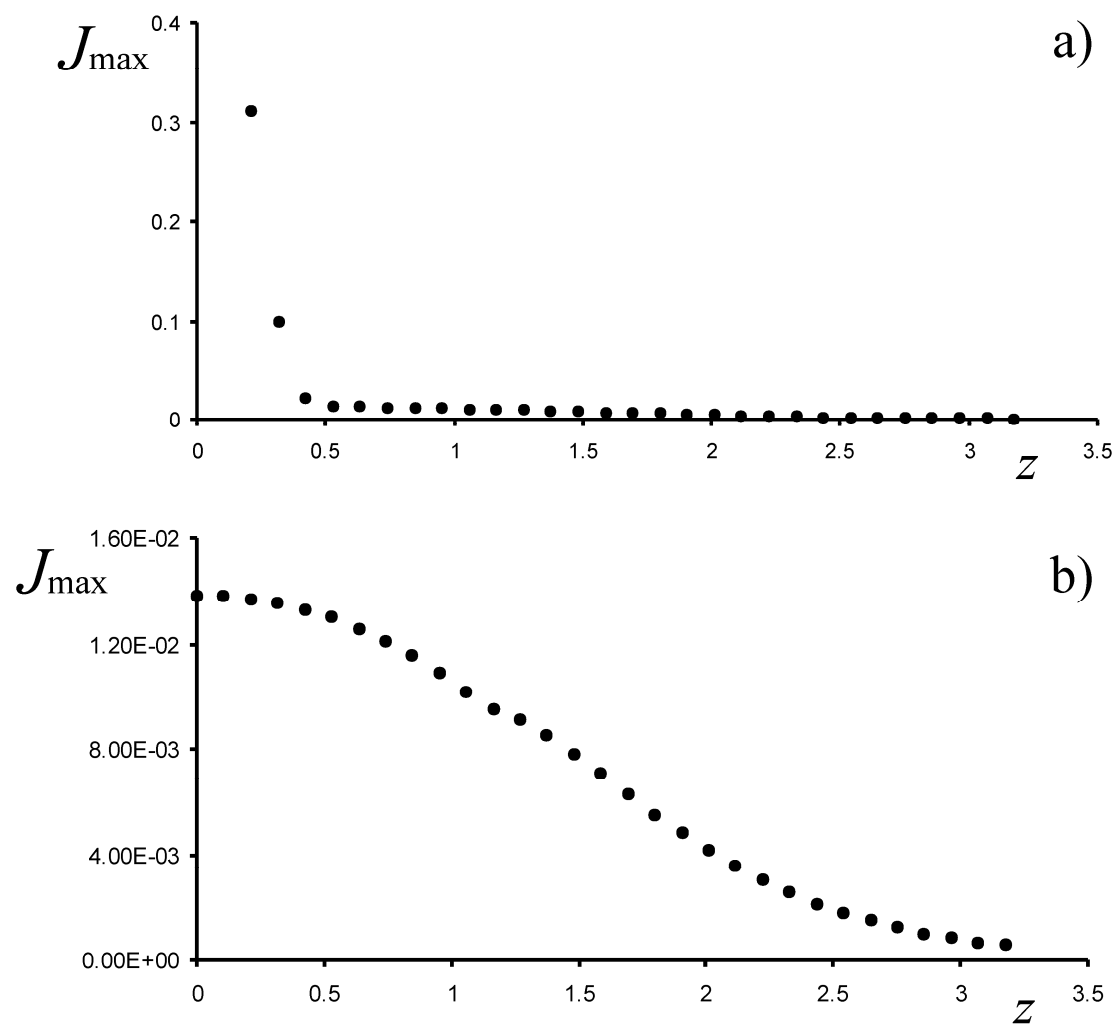

Figure 3. The dependence of the maximal value of the current density in the plotting plane $\left(J_{\max }\right)$ on the height above the molecular plane of $\mathrm{Na}_{6}$. Current densities were calculated by means of the CTOCD-DZ method using: a) 6-31G* and b) LANL2DZ basis set with the LANL2DZ effective core potential. The values of $J_{\max }$ are in a.u. whereas the distances are in $\AA$. 
a)

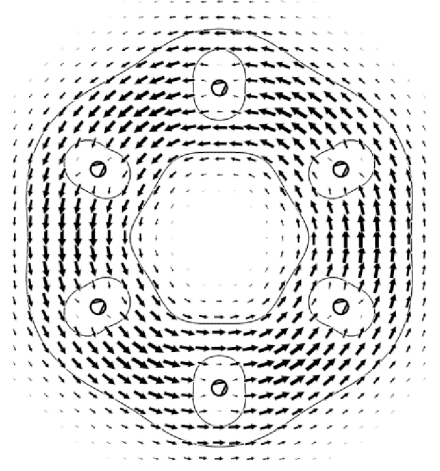

b)

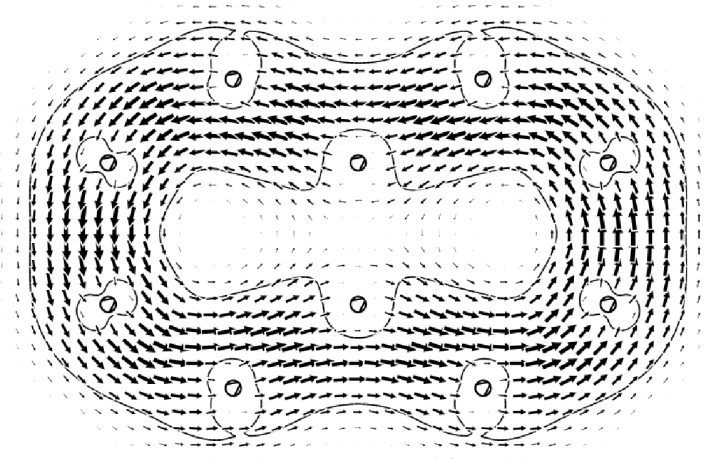

c)

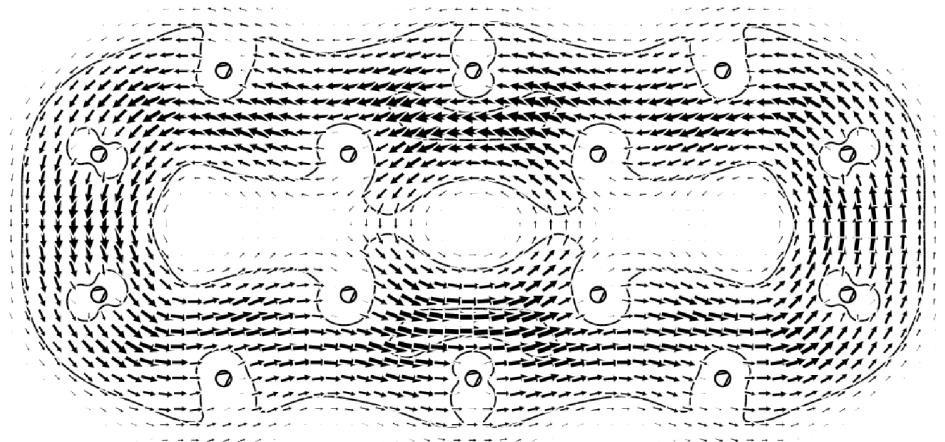

d)

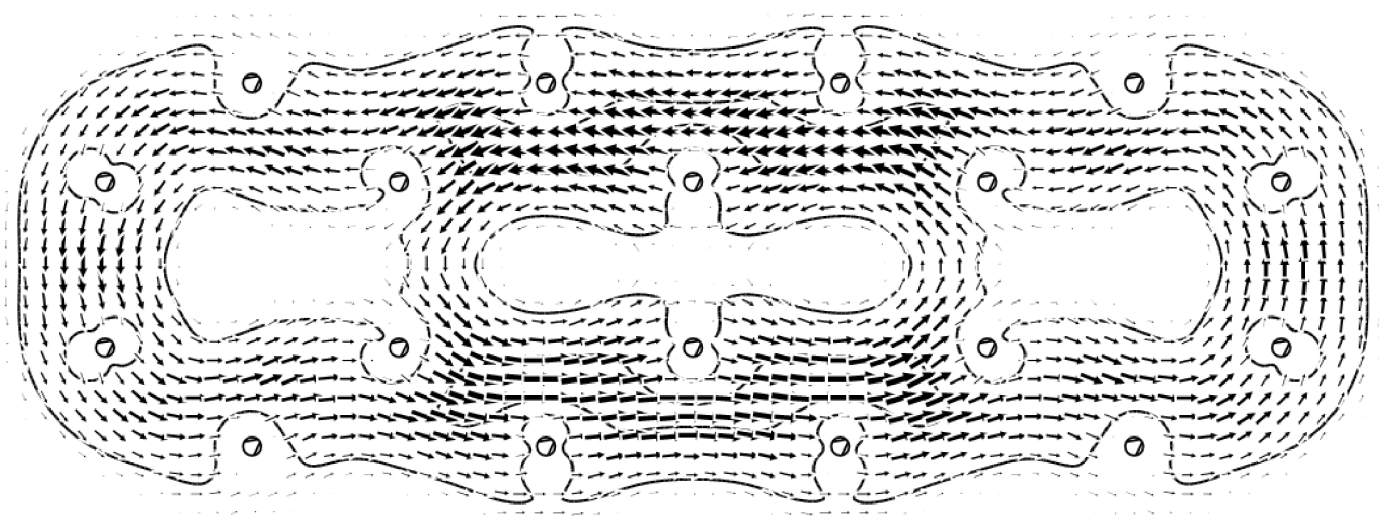

e)

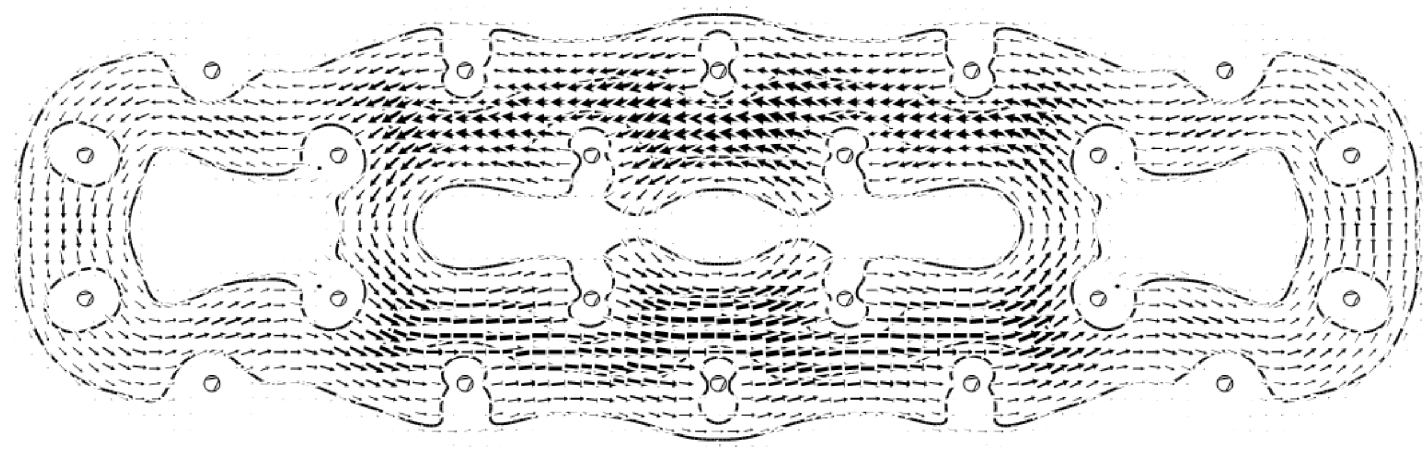


Figure 4. Total current density maps calculated using the 6-31G basis set: a) $\mathrm{Na}_{6}$ b) $\mathrm{Na}_{10}$ c) $\mathrm{Na}_{14}$ d) $\mathrm{Na}_{18}$ e) $\mathrm{Na}_{22}$. For details see the text. 
a)

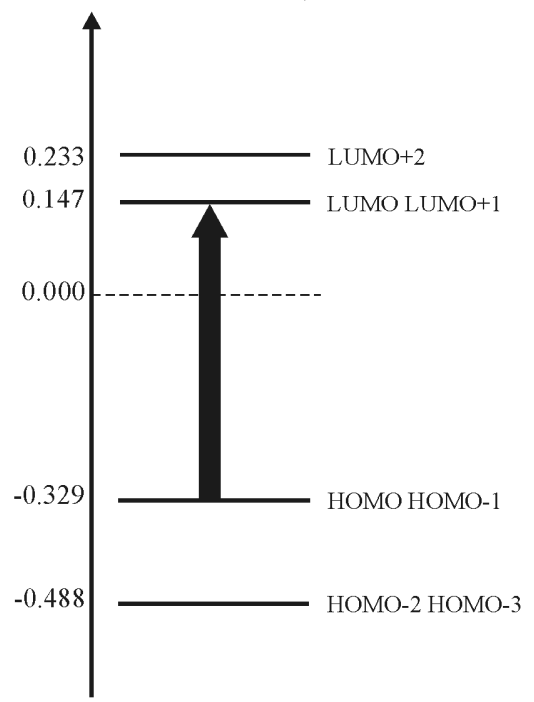

b)

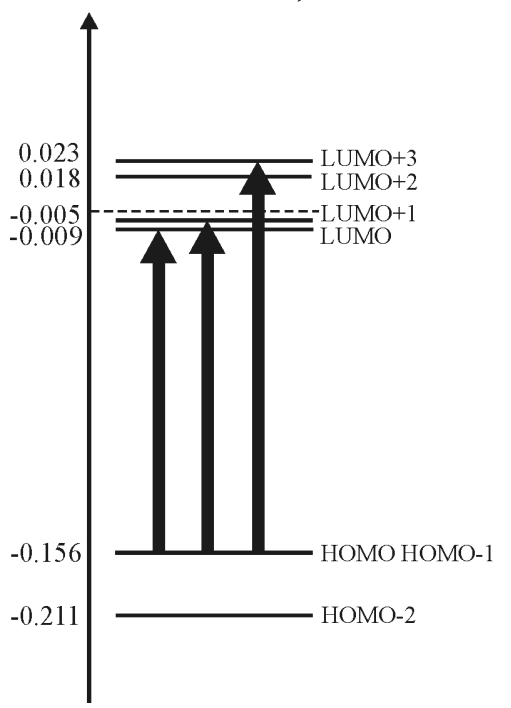

Figure 5. Orbital energy level diagram for: a) benzene and b) $\mathrm{Na}_{6}$. The energies (in a.u.) are obtained at the HF/6-31G* level. Only the transitions that significantly contribute to the induced current density are shown. Black arrows represent translational (diatropic) transitions and the width of arrows reflects the relative magnitude of the contribution of the underlined transition (for details see Ref. 29). 
a)

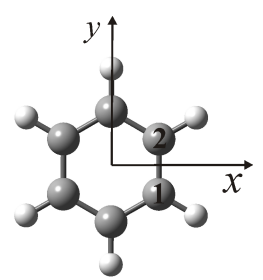

$\left.\mathrm{b}_{1}\right)$

$\left.\mathrm{b}_{2}\right)$

$\left.b_{3}\right)$
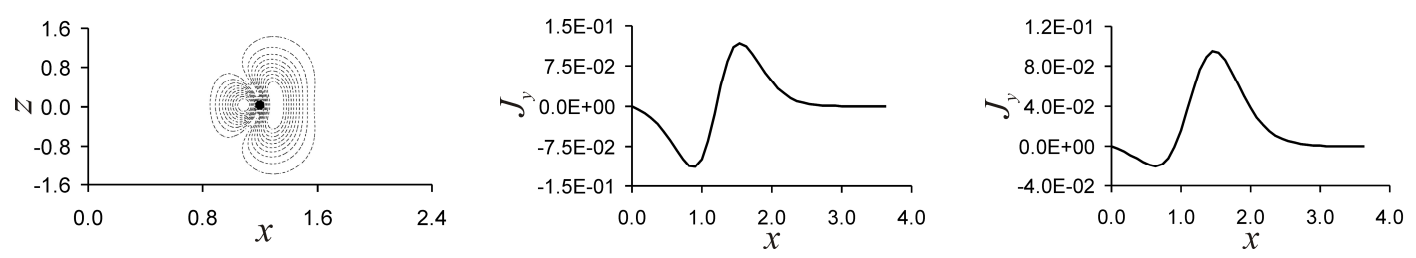

$\left.\mathrm{c}_{1}\right)$

$\left.\mathrm{c}_{2}\right)$

$\left.\mathrm{c}_{3}\right)$
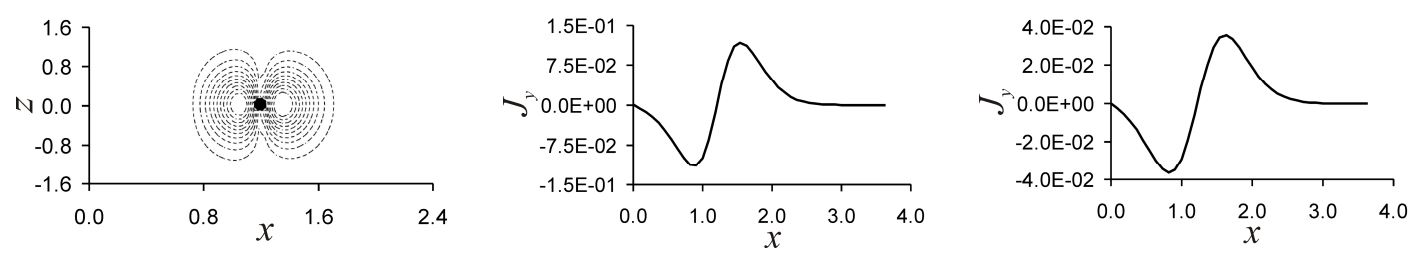

$\left.\mathrm{d}_{1}\right)$

$\left.\mathrm{d}_{2}\right)$

$\left.\mathrm{d}_{3}\right)$
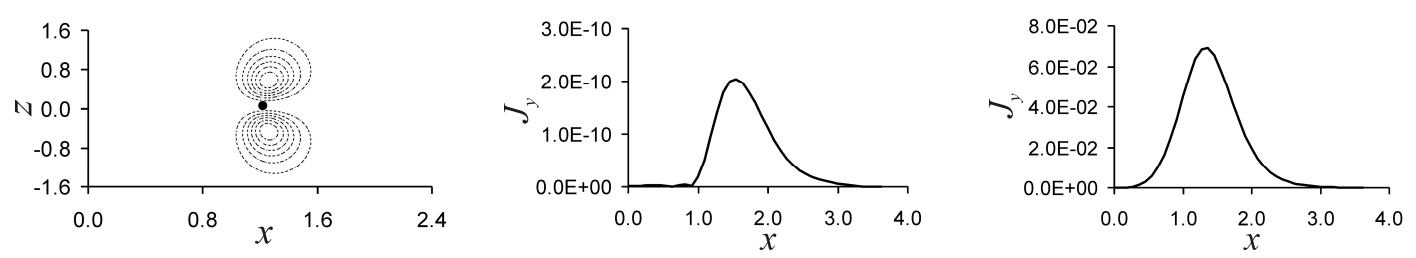

Figure 6. a) The coordinate system used in this figure; the benzene ring lies in the $x y$ plane; the ring centre is at the origin; the centre of the $\mathrm{C} 1-\mathrm{C} 2$ bond is at $(x, y)=(1.21$, 0.00) $\AA$. The current density cross sections calculated at the CTOCD-DZ/HF/6$31 \mathrm{G}^{*} / / \mathrm{B} 3 \mathrm{LYP} / 6-311+\mathrm{G}^{*}$ level for a plane perpendicular to the $\mathrm{C} 1-\mathrm{C} 2$ bond in benzene and passing through the bond centre: $b_{1}$ ) all electrons, $\left.c_{1}\right) \sigma$-electrons, $\left.d_{1}\right) \pi$ electrons. Dashed lines show the modulus of the current density. The symbol denotes the position of the centre of the $\mathrm{C} 1-\mathrm{C} 2$ bond in the $x z$-plane. The dependence of the $J_{y}$ component of the total current density vector calculated in the molecular plane on the distance from the centre of the benzene ring along the $x$-axis: $b_{2}$ ) all electrons, $\left.\mathrm{c}_{2}\right) \sigma$-electrons and $\left.\mathrm{d}_{2}\right) \pi$-electrons. The dependence of the $J_{y}$ component of the total current density calculated $1 \mathrm{a}_{0}$ above the molecular plane on the distance from the centre of the benzene ring along the $x$-axis: $b_{3}$ ) all electrons, $\left.c_{3}\right) \sigma$-electrons and 
$\left.\mathrm{d}_{3}\right) \pi$-electrons. Positive (negative) values of $J_{y}$ correspond to a diatropic (paratropic) current. The values of $J_{y}$ are in a.u,. whereas the distances are in $\AA$. 
a)

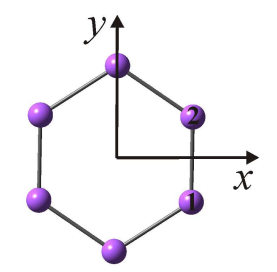

$b_{1}$ )

$b_{2}$ )

$\left.\mathrm{b}_{3}\right)$
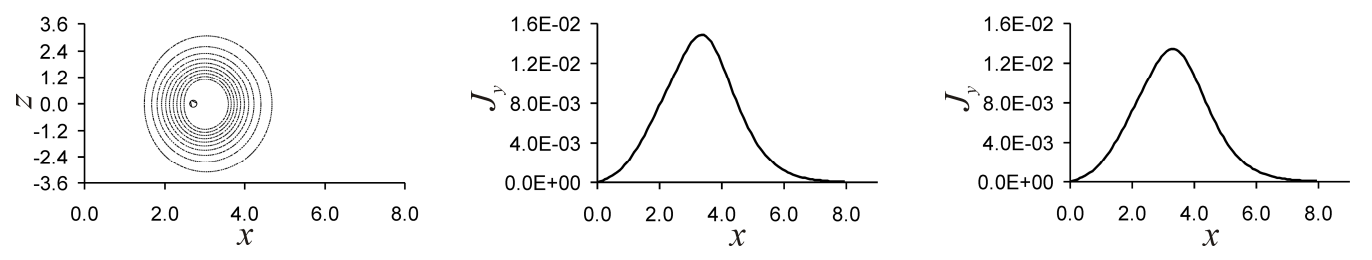

$\mathrm{c}_{1}$ )

$\mathrm{c}_{2}$ )

$\left.\mathrm{c}_{3}\right)$
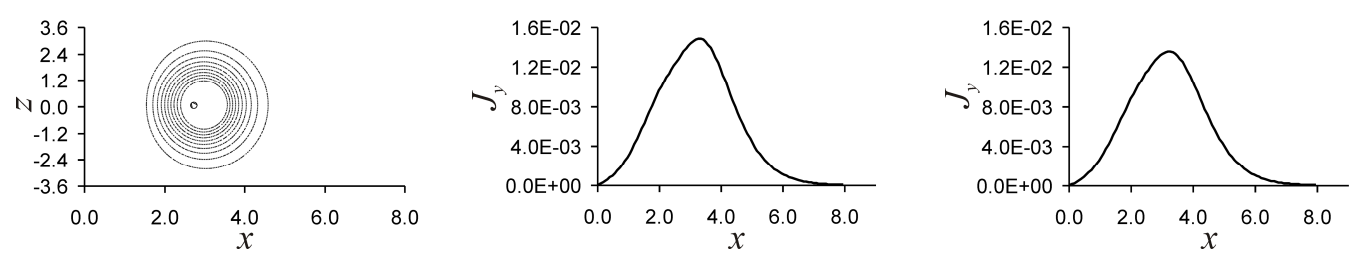

$\mathrm{d}_{1}$ )

$\mathrm{d}_{2}$ )

$\left.\mathrm{d}_{3}\right)$
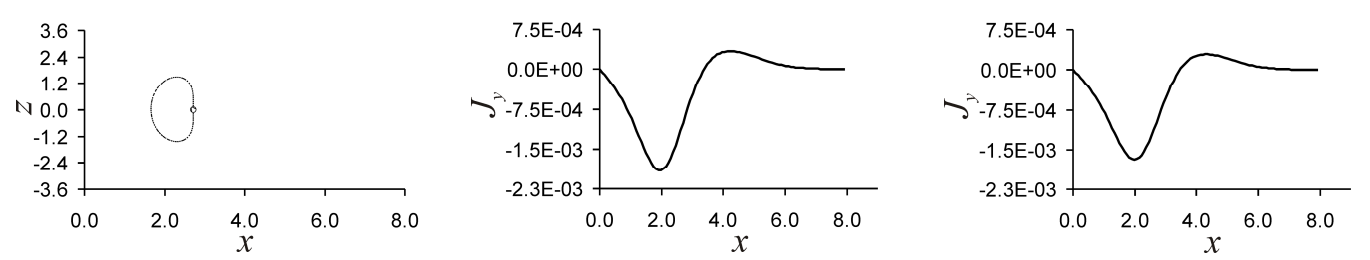

Figure 7. a) The coordinate system used in this figure; the $\mathrm{Na}_{6}$ ring lies in the $x y$ plane; the ring centre is at the origin; the centre of the Na1-Na2 bond is at $(x, y)=$ (2.72, 0.00) $\AA$. The current density cross sections calculated at the CTOCD-DZ/HF/6$31 \mathrm{G}^{*}$ level for a plane perpendicular to the $\mathrm{Na} 1-\mathrm{Na} 2$ bond in $\mathrm{Na}_{6}$ and passing through the bond centre: $b_{1}$ ) all electrons, $c_{1}$ ) HOMO pair, $d_{1}$ ) HOMO-2. Dashed lines show the modulus of the current density. The symbol $\circ$ denotes the position of the centre of the Na1-Na2 bond in the $x z$-plane. The dependence of the $J_{y}$ component of the current density vector in the molecular plane on the distance from the centre of $\mathrm{Na}_{6}$ ring along the $x$ - axis: $\mathrm{b}_{2}$ ) all electrons, $\mathrm{c}_{2}$ ) HOMO pair and $\mathrm{d}_{2}$ ) HOMO-2. The dependence of the $J_{y}$ component of the total current density $1 \mathrm{a}_{0}$ above the molecular plane on the 
distance from the centre of $\mathrm{Na}_{6}$ ring along the $x$ - axis: $\mathrm{b}_{3}$ ) all electrons, $\mathrm{c}_{3}$ ) HOMO pair and $d_{3}$ ) HOMO-2. See Figure 6 caption for other details. 


\section{Captions of tables and figures}

Table 1. NICS(0) and NICS(1) values of individual rings of Na-clusters depicted in Figure 1. NICS values were calculated at HF level using the STO-3G (I), 3-21G(II), 6-31G(III), 6-31G*(IV), 6-311+G*(V) basis set and the LANL2DZ basis set with effective core potential (VI).

Table 2. NICS(0) and NICS(1) values of individual rings of linear polyacenes analogous to Na-clusters depicted in Figure 1. NICS values were calculated at HF level using the STO-3G (I), 3-21G(II), 6-31G(III), 6-31G*(IV), 6-311+G*(V) basis set and the LANL2DZ basis set with effective core potential (VI). Geometries were optimized at the B3LYP/6-311+G* level.

Figure 1. The structures of Na-clusters: a) $\mathrm{Na}_{6}$ b) $\mathrm{Na}_{10}$ c) $\mathrm{Na}_{14}$ d) $\mathrm{Na}_{18}$ e) $\mathrm{Na}_{22}$. The geometries of Na-clusters were taken from the experimental crystallographic data. ${ }^{14}$ The capital letters denote individual six-membered rings used for the NICS calculations.

Figure 2. Current density maps of $\mathrm{Na}_{6}$ calculated using the $6-31 \mathrm{G}^{*}$ basis set: a) total current density in the molecular plane, b) HOMO pair contributions to the total current density presented in a), c) total current density $1 \mathrm{a}_{0}$ above the molecular plane with the arrows three times enlarged, d) HOMO pair contributions to the total current density presented in c).

Figure 3. The dependence of the maximal value of the current density in the plotting plane $\left(J_{\max }\right)$ on the height above the molecular plane of $\mathrm{Na}_{6}$. Current densities were calculated by means of the CTOCD-DZ method using: a) 6-31G* and b) LANL2DZ basis set with the LANL2DZ effective core potential. The values of $J_{\max }$ are in a.u. whereas the distances are in $\AA$.

Figure 4. Total current density maps calculated using the 6-31G basis set: a) $\mathrm{Na}_{6}$ b) $\mathrm{Na}_{10}$ c) $\mathrm{Na}_{14}$ d) $\mathrm{Na}_{18}$ e) $\mathrm{Na}_{22}$. For details see the text. 
Figure 5. Orbital energy level diagram for: a) benzene and b) $\mathrm{Na}_{6}$. The energies (in a.u.) are obtained at the $\mathrm{HF} / 6-31 \mathrm{G}^{*}$ level. Only the transitions that significantly contribute to the induced current density are shown. Black arrows represent translational (diatropic) transitions and the width of arrows reflects the relative magnitude of the contribution of the underlined transition (for details see Ref. 29).

Figure 6. a) The coordinate system used in this figure; the benzene ring lies in the $x y$ plane; the ring centre is at the origin; the centre of the C1-C2 bond is at $(x, y)=(1.21$, 0.00) $\AA$. The current density cross sections calculated at the CTOCD-DZ/HF/631G*//B3LYP/6-311+G* level for a plane perpendicular to the C1-C2 bond in benzene and passing through the bond centre: $b_{1}$ ) all electrons, $\left.c_{1}\right) \sigma$-electrons, $\left.d_{1}\right) \pi$ electrons. Dashed lines show the modulus of the current density. The symbol $\bullet$ denotes the position of the centre of the C1-C2 bond in the $x z$-plane. The dependence of the $J_{y}$ component of the total current density vector calculated in the molecular plane on the distance from the centre of the benzene ring along the $x$-axis: $b_{2}$ ) all electrons, $\mathrm{c}_{2}$ ) $\sigma$-electrons and $\mathrm{d}_{2}$ ) $\pi$-electrons. The dependence of the $J_{y}$ component of the total current density calculated $1 \mathrm{a}_{0}$ above the molecular plane on the distance from the centre of the benzene ring along the $x$-axis: $b_{3}$ ) all electrons, $\left.c_{3}\right) \sigma$-electrons and $\left.\mathrm{d}_{3}\right) \pi$-electrons. Positive (negative) values of $J_{y}$ correspond to a diatropic (paratropic) current. The values of $J_{y}$ are in a.u,. whereas the distances are in $\AA$.

Figure 7. a) The coordinate system used in this figure; the $\mathrm{Na}_{6}$ ring lies in the $x y$ plane; the ring centre is at the origin; the centre of the Na1-Na2 bond is at $(x, y)=$ $(2.72,0.00) \AA$. The current density cross sections calculated at the CTOCD-DZ/HF/6$31 \mathrm{G}^{*}$ level for a plane perpendicular to the $\mathrm{Na} 1-\mathrm{Na} 2$ bond in $\mathrm{Na}_{6}$ and passing through the bond centre: $b_{1}$ ) all electrons, $c_{1}$ ) HOMO pair, $d_{1}$ ) HOMO-2. Dashed lines show the modulus of the current density. The symbol $\circ$ denotes the position of the centre of the Na1-Na2 bond in the $x z$-plane. The dependence of the $J_{y}$ component of the current density vector in the molecular plane on the distance from the centre of $\mathrm{Na}_{6}$ ring along the $x$ - axis: $b_{2}$ ) all electrons, $c_{2}$ ) HOMO pair and $d_{2}$ ) HOMO-2. The dependence of the $J_{y}$ component of the total current density $1 \mathrm{a}_{0}$ above the molecular plane on the distance from the centre of $\mathrm{Na}_{6}$ ring along the $x$ - axis: $\mathrm{b}_{3}$ ) all electrons, $\mathrm{c}_{3}$ ) HOMO pair and $d_{3}$ ) HOMO-2. See Figure 6 caption for other details. 